II dibattito intorno al volgare antico tra

Leonardo Bruni e Flavio

Biondo sullo sfondo della cognizione linguistica di Dante

\section{Roger Schöntag}

Friedrich-Alexander-Universität Erlangen-Nürnberg, Germania
20I7, Vol. 5I(3) 553-572

(C) The Author(s) 2017

Reprints and permissions: sagepub.co.uk/journalsPermissions.nav DOI: $10.1177 / 00145858177 \mid 1685$ journals.sagepub.com/home/foi

(SAGE

\begin{abstract}
La nozione linguistica "latino volgare" e l'idea della diversificazione interna del latino risale a un dibattito nell'anticamera di papa Eugenio IV nel I435. Leonardo Bruni e Flavio Biondo esposero le loro convinzioni sul latino nell'antichità romana in due brevi trattati.

L'obiettivo del presente saggio è di ritrarre la discussione tra Bruni e Biondo e di esporre in quale maniera gli umanisti attingono alla teoria dantesca, anche se i due eruditi probabilmente non hanno avuto conoscenza diretta del trattato De vulgari eloquentia $(D V E)$, scritto che rappresenta, considerando il latino, un punto di vista tipicamente medievale (grammatica vs. vulgare, ars vs. natura). La nuova idea, il latino come lingua viva che cambia e si modifica, emerge in questo contesto storico.
\end{abstract}

\title{
Parole chiave
}

Flavio Biondo, Leonardo Bruni, Dante, latino, latino volgare, questione della lingua

\section{Introduzione}

Una delle nozioni principali della linguistica moderna riguardo alle lingue neolatine è che l'italiano, il francese, lo spagnolo e le altre lingue romanze hanno la loro origine nel latino volgare e non nel latino scritto, cioè classico. Indipendentemente dalla discussione riguardo alla datazione e al carattere di questo latino volgare, strutturalmente diverso dalla varietà cosiddetta classica, è communis opinio che la base dell'italiano odierno sia una varietà orale del latino e che questo latino parlato

\footnotetext{
Autore corrispondente:

Roger Schöntag, Institut für Romanistik, Friedrich-Alexander-Universität Erlangen-Nürnberg, Bismarckstraße I, 91054 Erlangen, Germania.

Email: Roger_schoentag@yahoo.de
} 
abbia lasciato anche tracce significative in alcuni documenti scritti (iscrizioni del latino tardo, certi autori e generi letterari, letteratura d'uso/letteratura di consumo ecc.).

La nozione e il concetto di latino volgare nel senso linguistico moderno risalgono a Hugo Schuchardt (1842-1927) e al suo capolavoro sul vocalismo, Der Vokalismus des Vulgärlateins (1866), basato sull'opera del fondatore della romanistica Friedrich Diez (1794-1876) Volkslatein (cfr. Diez, 1870: 6), traduzione diretta del vulgaris sermo di Marco Tullio Cicerone (Acad. I, 5). ${ }^{1}$

Nel medioevo, ossia all'epoca di Dante, il latino era considerato una lingua artificiale e invariabile (vedi infra cap. 2) e fu solo durante il Quattrocento e il Cinquecento che gli eruditi rinascimentali cominciarono a intuire che la lingua latina avesse i caratteri di una lingua eterogenea, con una dimensione storica alla stregua di ogni altra lingua. ${ }^{2}$

Il presente contributo vuole evidenziare in che modo nacque quest'idea del latino come lingua viva, cioè variabile, cercando altresì di delineare come il concetto del latino volgare si è sviluppato in senso moderno.

Con il sorgere della storiografia, nell'ambito di un interesse generale per gli scritti originali dei Romani e dei Greci, si svolse una famosa discussione tra Flavio Biondo (1392-1463) e Leonardo Bruni (1370-1444) sulla situazione linguistica nell'antichità romana. Questo dibattito dell'anno 1435 nell'anticamera del papa ebbe ripercussioni in due trattati (vedi infra cap. 3) che furono fonte di una controversia tra gli eruditi per più di un secolo.

L'idea della variabilità della lingua in generale non è però concepibile senza le esposizioni di Dante Alighieri (1265-1321) sul vulgare (cioè l'italiano), soprattutto nel suo trattato De vulgari eloquentia, che pertanto costituisce la base imprescindibile di questa ricerca. ${ }^{3}$

In modo da evidenziare più chiaramente l'origine del concetto linguistico della variabilità della lingua e della variabilità del latino in particolare, abbiamo deciso di applicare, accanto alla "diglossia" di Ferguson (1959), le moderne nozioni della sociolinguistica, soprattutto quelle fondate sulla teoria del "diasistema" (variazione diatopica, diastratica e diafasica) di Eugenio Coseriu (1973), ulteriormente sviluppata ed elaborata da Gaetano Berruto $(1987,2003)$ e da Peter Koch e Wulf Oesterreicher $(1985,2011)$.

\section{Volgare e latino nel De vulgari eloquentia di Dante}

Il grande poeta Dante Alighieri (1265-1321), che segna un inizio importante per tanti aspetti nella letteratura italiana, è anche il primo da citare quando si parla di preistoria linguistica. Sulla base del suo trattato apologetico De vulgari eloquentia (1303/1304), riscoperto (1515) e tradotto (1529) in italiano da Gian Giorgio Trissino (1478-1550), si può rilevare molto bene come Dante fosse contemporaneamente radicato nel patrimonio intellettuale del medioevo e dell'età moderna. ${ }^{4}$ Obiettivo principale della suddetta doctrina è certamente la ricerca della forma ideale della lingua italiana per la produzione letteraria (illustre, cardinale, aulicum et curiale 
vulgare, DVE I, 16, 6), però il poeta quasi en passant illustra anche il suo pensiero sulla differenza tra il latino e il volgare, cioè l'italiano. ${ }^{5}$

Il latino - e Dante si riferisce anzitutto al latino della sua epoca - è per lui una lingua secondaria (locutio secundaria) da apprendere, ${ }^{6}$ una disciplina che richiede molto tempo e assiduità (per spatium temporis et studii assiduitatem), ragion per cui la competenza linguistica di questo idioma non è molto diffusa.

Est et inde alia locutio secundaria nobis, quam Romani gramaticam vocaverunt. Hanc quidem secundariam greci habent et alii, sed non omnes: ad habitum vero huius pauci perveniunt, quia non nisi per spatium temporis et studii assiduitatem regulamur et doctrinamur in illa. (DVE I, 3)

Il motivo per il quale il latino si chiama gramatica (sic!) è dato prima facie con la formulazione Romani vocaverunt, però Dante stesso offre anche altri argomenti più importanti. Il latino è una lingua artificiale (artificialis, DVE I, 1, 4) e conseguentemente vi sono anche inventori (inventores gramatice, DVE I, 9, 11) di questo idioma. $^{7}$

La caratteristica cruciale è tuttavia che il latino è una lingua avente delle regole, un'attribuzione anzitutto ex silentio, dato che Dante $(D V E \mathrm{I}, 1,2)$ definisce il volgare come lingua sine omni regula - concetto che solo successivamente nel discorso diventa un po' più esplicito (fuerit regulata, DVE I, 9, 11).

Il poeta mostra inoltre le diverse funzioni del latino, che consentono l'accesso alle autorità letterarie (autoritates), alle gesta degli antenati (gesta), e la possibilità di comunicare fra genti diverse che non vivono nello stesso luogo (locorum diversitas).

Adinvenerunt ergo illam ne, propter variationem sermonis arbitrio singularium fluitantis, vel nullo modo vel saltim imperfecte antiquorum actingeremus autoritates et gesta, sive illorum quos a nobis locorum diversitas facit esse diversos. (DVE I, 9, 11)

Tutto ciò è possibile, perché il latino ha la proprietà di essere invariabile (inalterabilis), più precisamente invariabile nello spazio e nel tempo (diversibus temporibus atque locis, DVE I, 9, 11). Con questa caratterizzazione del latino, Dante descrive anche le funzioni principali di una lingua scritta fine a se stessa, che serve da memoria collettiva di una società e che deve garantire la comunicazione attraverso lo spazio e il tempo.

Quando applichiamo la terminologia moderna della diasistematica, possiamo costatare che Dante intende il latino come una lingua diacronicamente invariabile, negando così ogni mutamento linguistico (nec variabilis esse potest, DVE I, 9, 11). Riguardo alla variabilità sincronica, Dante dichiara esplicitamente, come già detto, che il latino serve anche per comunicare con persone che si trovano in un luogo diverso, fatto che si può interpretare sia in modo funzionale (comunicazione a distanza), sia come un'invariabilità diatopica del latino. Tuttavia, come vedremo, la caratteristica dell'invariabilità concernente le singole dimensioni della diatopica, 
diastratica e diafasica si può solamente dedurre ex negativo dalla variabilità della vulgaris locutio.

L'omogeneità e l'invariabilità del latino, e in genere di una lingua erudita (secondaria), ${ }^{8}$ risalgono alla tradizione scolastica. La grammatica era la prima parte nel curriculum di un futuro erudito che doveva studiare le septem artes liberales, dunque il trivium e il quadrivium. In tal modo il latino era sia uno strumento per lo studio, sia la prima disciplina. Il latino, appreso sulla base delle opere di Donato (Aelius Donatus, IV sec.: Ars maior, Ars minor) e Prisciano (Priscianus Caesariensis, fine del V sec.: Institutiones grammaticae), era una lingua regolata con una funzione chiara in una società diglossica. Nell'ambito dello studio, il latino così era diventato una lingua che non poteva cambiare sostanzialmente, perché era lo strumento (Conv. $\mathrm{I}, 11,11)$ imprescindibile per le cognizioni filosofiche e teologiche. Più tardi i modisti (modistae) fecero una distinzione tra grammatica (nel senso di lezioni del latino) e grammatica speculativa nel senso di una teoria sulla natura della lingua, ma il criterio dell'inalterabilità rimase. ${ }^{9}$

Eppure l'idea dell'invariabilità di una lingua, che era regolata, non era nuova, poiché già Quintiliano (Marcus Fabius Quintilianus, ca. 35-95 d.C.) aveva fatto una distinzione tra il latino e il modo di parlare secondo la grammatica.

Quare mihi non invenuste dici videtur aliud esse Latine, aliud grammatice loqui.

(Quint., Inst. Orat. I, 6, 27)

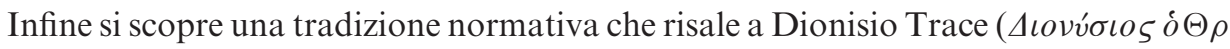
$\alpha \xi$, II sec. a.C.: $\left.T \varepsilon^{\prime} \chi \nu \eta \gamma \rho \alpha \mu \mu \alpha \tau \iota \kappa \eta^{\prime}\right)$, il primo grammatico greco, e a Varrone (Marcus Terentius Varro, 116-27 a.C.) che ne modificò leggermente il modello per adattarlo al latino. Quest'ultimo nella sua opera De lingua latina tenta di dimostrare come sarebbe possibile conciliare l'uso naturale della lingua (consuetudo) con i principi grammaticali dell'anomalia (anomalia) e dell'analogia (analogia). ${ }^{10}$ Per Varrone l'innovazione (declinatio voluntaria), che è sia un impulso naturale della consuetudo, sia un fattore importante per la produzione letteraria, è lecita, a patto che la nuova parola venga sottomessa alla declinatio naturalis, ${ }^{11}$ cioè alla flessione corretta (Jungen e Lohnstein, 2007: 60). ${ }^{12}$ Nella tradizione grammaticale e retorica si osservano la genesi di una prassi di normalizzazione e standardizzazione e l'origine di una differenziazione tra una varietà di lingua che segue l'uso e un'altra varietà che viene regolata e fissata. Quest'ultima varietà è visibile nelle opere dei "buoni” autori (auctores), il cui uso della lingua riflette le regole che erano state riscoperte e sistematizzate in un modello (ars grammatica) dai grammatici, insegnata e diffusa nelle scholae (Poccetti et al., 2005: 416-417). ${ }^{13}$

Dopo secoli di trasmissione del modello di una lingua invariabile, ${ }^{14}$ nel quale l'invariabilità stessa ha anche un aspetto di superiorità filosofica (cfr. Cheneval, 1996: 128-130), Dante non aveva nessuna ragione di mettere in dubbio le caratteristiche di questa lingua sacra, soprattutto considerando i vantaggi menzionati. 
Riguardo alla lingua volgare, invece, Dante dimostra attraverso numerosi particolari ed esempi specifici di avere una concezione molto chiara della variabilità della lingua o più esattamente di una lingua naturale. ${ }^{15}$ Dato che l'eterogeneità del volgare è già stata oggetto di tanti studi, ${ }^{16}$ possiamo limitarci a enumerarne gli aspetti più importanti e ad applicare la terminologia moderna.

Contrariamente al latino il vulgaris è naturale (tum quia naturalis est nobis, DVE I, $1,4)$, una lingua che non si deve studiare, visto che si impara nell'infanzia (maternam locutionem, DVE I, 6, 2), sia dalle persone circostanti (ab assistentibus, DVE I, 1, 2) sia dalla nutrice (nutricem imitantes, $D V E \mathrm{I}, 1,2$ ). Il volgare ha dunque lo stato di una locutio primaria, un termine che Dante non usa, ${ }^{17}$ che si può però dedurre dalla denominazione che usa per il latino (locutio secundaria). Inoltre il volgare è una lingua senza regole (sine omni regula, DVE I, 1, 2). Con questa caratterizzazione Dante si riferisce a un'opposizione importante nel mondo intellettuale del medioevo, cioè ars vs. natura (cfr. Ellena, 2011: 61).

L'architettura del volgare emerge dalle sue osservazioni sull'origine della lingua e sulla diversificazione linguistica nelle diverse regioni del mondo e d'Italia - sempre sullo sfondo della ricerca sul vulgare illustre.

Nel campo della distribuzione tipologica ${ }^{18}$ delle lingue europee Dante fornisce anche una precisa descrizione diatopica del volgare in Italia (DVE I, 10, 4-7). ${ }^{19}$ Tuttavia, la sua dimensione diatopica non è solo limitata a un'enumerazione dei dialetti, ${ }^{20}$ ma considera anche una differenziazione tra dialetto nell'ambito di un'arealità più vasta e un vernacolo con una diffusione più limitata, dunque in una città o in un suo quartiere (istarum variationum in se ipsa variatur, DVE I, 9, 4). ${ }^{21}$ In relazione al modo di parlare nei diversi quartieri, sembra esserci un'intersezione tra la dimensione diatopica, diastratica e diatecnica. ${ }^{22}$ Una diversità diatecnica può essere anche riscontrata nella descrizione degli operai che partecipano alla costruzione della torre di Babele; ${ }^{23}$ la variabilità difasica e diastratica si rivela nel modo di parlare a seconda della loro occupazione. ${ }^{24}$

La dimensione diamesica vale solo per la prospettiva propria di Dante, che intende il latino come parte integrativa del suo mondo linguistico. Dalla prospettiva moderna, invece, la situazione all'epoca di Dante è una diglossia classica con una distribuzione funzionale tra scritto e parlato con due lingue parenti. Diamesico in sensu strictu si riferisce invece alla distribuzione orale e scritturale all'interno di una lingua. ${ }^{25}$

Il motivo della variabilità diacronica, dunque il mutamento stesso della lingua, per Dante è dovuto all'instabilità della natura degli uomini. Il fatto che l'uomo è un instabilissimum atque variabilissimum animal (DVE I, 9, 6) è presentato come conditio humana. È notevole che Dante distingua da una parte un mutamento leggero come quello tra il parlare dei vecchi e dei giovani ( $D V E$ I, 9, 8), e dall'altra un mutamento più profondo come tra il parlare in un'epoca remota (a Pavia) e il parlare contemporaneo (vetustimus Papiensis vs. modernis Papiensis, DVE I, 9, 6). ${ }^{26}$ Da una prospettiva moderna si potrebbe costatare che Dante adduce un criterio sociolinguistico, visto che la variabilità della lingua (nec durabilis nec continua esse 
potest, DVE I, 9, 6) è collegata alla variabilità dei costumi e tradizioni (mores et habitus) - la lingua come fenomeno del terzo tipo. ${ }^{27}$

$\mathrm{Ci}$ sono dunque due punti decisivi per la presente analisi: in primo luogo il fatto che Dante osserva un legame tra il latino e il volgare (le lingue romanze/ l'italiano, cfr. $D V E$ I, 8, 5), pur tuttavia non indicando la genealogia di un mutamento con un processo diacronico linguistico, in quanto il latino come gramatica rimane inalterabilis. Il secondo ydioma tripharium non è reso esplicito, il postulato di un vulgare latinum come espresso da Coseriu e Meisterfeld (2003: 158) non è plausibile. ${ }^{28}$

In secondo luogo, anche se Dante ha una consapevolezza del legame tra il latino e le lingue romanze, non ha potuto capire o trasferire la variabilità del volgare all'epoca anteriore, cioè alla situazione linguistica nell'antichità. Per questo la variabilità della lingua descritta nel passo spesso citato da Orazio (Quintus Horatius Flaccus, 65-27 a.C.) ${ }^{29}$ è per Dante solo interpretabile come variabilità di un volgare qualsiasi, mai come variabilità della lingua latina.

In rapporto al latino Dante è dunque radicato in una prospettiva medievale tradizionale, per il volgare, la lingua naturale, invece batte nuove strade.

\section{Volgare e latino negli scritti di Leonardo Bruni e Flavio Biondo}

L'interesse filologico documentato per la situazione linguistica dell'antichità cominciò con una disputa nell'anticamera di papa Eugenio IV (1431-1447) a Firenze ${ }^{30}$ nell'anno 1435. I segretari papali (apostolici secretari) Antonio Loschi (1365-1441), Cencio Romano (1390-1445), Andrea Fiocchi (1400-1452), Poggio Bracciolini (1380-1459), Flavio Biondo (1392-1463) e Leonardo Bruni (1370-1444) discussero il problema della lingua materna degli antichi Romani (cfr. Marchiò, 2008: 17).§

Come abbiamo dimostrato nel capitolo precedente, l'opinione più diffusa all'epoca era che gli antichi parlassero quasi come scrivevano, dunque nell'idioma regolato, la cosiddetta grammatica; si affermò però anche l'idea che parlassero una sorta di volgare. ${ }^{31}$

I due primi e più importanti protagonisti della discussione furono Flavio Biondo (Flavius Blondus), colui che ha descritto la suddetta situazione nell'anticamera pontificia, e Leonardo Bruni (Leonardus Aretinus), ${ }^{32}$ che redasse una risposta abbastanza breve agli argomenti di Biondo. ${ }^{33}$ Entrambi furono tra i più eruditi del proprio tempo, con conoscenze filologiche e storiche straordinarie che si riflettono nelle loro opere. Mentre Bruni, che si occupava anche della storia italiana, era piuttosto un importante traduttore (dal latino e dal greco) e filologo, Biondo si dedicava soprattutto alla storia e agli studi antiquari. ${ }^{34}$

\section{I. Leonardo Bruni}

In un trattato nella forma di una lettera con il titolo Leonardus Flavio Foroliviensi S. Quaerit an vulgus et literati eodem modo per Terentii Tulliique tempora Romae 
locuti sint (1435), Bruni espone al suo interlocutore Flavio Biondo di Forlì la sua concezione linguistica, che era la più diffusa nella sua epoca.

Secondo Bruni - come già per Dante - esistono principalmente due lingue in Italia, ciò̀ il latino e il volgare. L'aspetto nuovo, che risultava della discussione summenzionata, era l'interesse per gli antichi e il loro modo di parlare (e di scrivere).

Secondo le sue esposizioni nella lettera citata, esistevano per lui due lingue diverse, cioè la lingua degli eruditi (docti, litterati oratores, nobiles) e della letteratura da una parte e la lingua parlata (lingua vulgare) dall'altra. Anche se Bruni non parla esplicitamente di grammatica, sebbene di lingua litterata o di sermo litteratus (Br. 2), intende questo concetto sicuramente nella stessa maniera di Dante. ${ }^{35}$ Inoltre la sua terminologia mostra che per lui il latino e la letteratura latina formano una sorta di unità (latine ac litterate loqui) o, in altre parole, per lui le due nozioni sono quasi tautologiche (cfr. Tavoni, 1984: 32; Marazzini, 2013: 33-39).

Questio nostra in eo consistit, quod tu apud veteres unum eumdemque fuisse sermonem omnium putas, nec alium vulgarem, alium litteratum. Ego autem, ut nunc est, sic etiam tunc distinctam fuisse vulgarem linguam a litterata existimo. (Br. 2)

Bruni motiva questa prospettiva con l'argomento che il popolo semplice (illitterati, indocti, vulgus, populus) non era in grado di capire la grammatica, perché essa richiedeva uno studio notevole affinché venisse correttamente appresa. Bruni adduce differenze semantiche, sintattiche e il problema della flessione per illustrare le differenze tra il latino letterario e il latino volgare. Di conseguenza il popolo sarebbe stato capace di comprendere le commedie di Plauto (Titus Maccius Plautus, ca. 250-184 a.C.) o Terenzio (Publius Terentius Afer, ca. 195/185-159 a.C.) solo parzialmente e in modo passivo. D'altro canto gli oratori usavano la grammatica sia nella comunicazione con altri eruditi, sia nelle rielaborazioni scritte dei propri discorsi. ${ }^{36}$

Bruni applica la situazione della propria epoca, caratterizzata da una diglossia (latino e italiano), ${ }^{37}$ all'epoca degli antichi Romani, quasi senza apportare modifiche e accentuando la dicotomia notevole ars vs. natura. La questione del modo in cui il volgare antico si trasforma in volgare contemporaneo non lo interessa, oppure almeno non viene esplicitata nel suo lavoro. ${ }^{38}$ Lo sviluppo linguistico non è al centro della sua attenzione, perché la sua prospettiva è quella di un grammatico che difende il primato del latino, dunque la grammatica.

In confronto a Dante l'attenzione verso la situazione linguistica dell'antichità è nuova. Inoltre Bruni (al contrario di Dante) è molto chiaro nelle sue spiegazioni sulla distribuzione delle lingue. Bruni è radicato nel concetto tradizionale della grammatica, perciò le sue descrizioni sulla variabilità linguistica del volgare sono meno dettagliate di quelle di Dante. Mentre Dante era alla ricerca di un italiano degno della lingua letteraria, cioè un vulgare illustre, e per questo costretto a riflettere a fondo sulle caratteristiche del volgare, Bruni vede se stesso come difensore della lingua latina (cfr. umanesimo latino). 


\section{Leonardo Bruni}

Leonardus Flavio Foroliviensi S. Quaerit an vulgus et literati eodem modo per Terentii Tuliique tempora

Romae locuti sint (1435)

Ego autem, ut muc est etiam, sic tumc distinctam fuisse vulgarem lingıam a literata existimo.

(Br. 2)

l'antichità

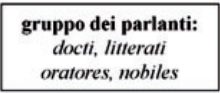

oratores, nobiles
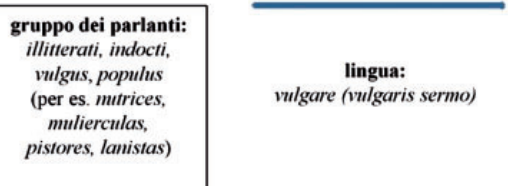

$\Rightarrow$ situazione diglossica con due lingue
1435

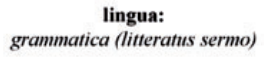

lingua:

grammatica

$?$

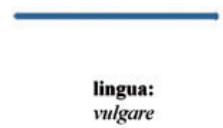

Figura I. La situazione linguistica secondo Leonardo Bruni.

Per illustrare le idee fondamentali di Bruni sulla distribuzione del latino e del volgare nell'antichità e nella sua epoca si rimanda al grafico seguente:

Nella terminologia moderna si può constatare che per Bruni le differenze tra il latino e il volgare sono radicate a livello diastratico e diafasico. L'uso delle due lingue è esplicitamente legato a un certo gruppo dei parlanti, ossia il latino viene utilizzato da una classe distinta per discendenza (nobiles) e istruzione (docti, litterati, oratores), mentre il volgare è diffuso tra certe professioni del ceto inferiore (pistores, lanistas) e tra gli illetterati e gli incolti (indocti, illitterati). Diafasicamente il latino letterario, che secondo Bruni è legato alla classe superiore (livello diastratico), ${ }^{39}$ si distingue dal volgare per una struttura grammaticale più complessa e un lessico particolare (cfr. Br. 32, 41). ${ }^{40}$

Bruni non s'interessa al mutamento linguistico del volgare e ritiene impossibile uno sviluppo del latino (da cui il punto interrogativo nel grafico precedente), mentre è ovvio che egli comprenda la situazione contemporanea con una lingua elaborata e invariabile (il latino) e una lingua per l'odierna comunicazione orale (il volgare) mutatis mutandis, allo stesso modo come avveniva nell'antichità.

Praestantes igitur homines oratorem Latine litterateque concionantem praeclare intelligebant, pistores vero et lanistae et huiusmodi turba sic intelligebant oratoris verba ut nunc intelligunt Missarum solemnia. (Br. 14)

In altre parole, Bruni sostiene l'idea che all'epoca degli antichi Romani esistessero due lingue diverse tra loro, però reciprocamente comprensibili, almeno in parte: una 
situazione paragonabile a quella contemporanea nell'Italia del Rinascimento (appunto ut nunc intelligunt Missarum, "come il popolo di oggi capisce la messa").

\subsection{Flavio Biondo}

L'aspetto nuovo del concetto linguistico di Flavio Biondo, esposto nella sua lettera De verbis Romanae locutionis Blondi ad Leonardum Aretinum (1435), consiste nell'idea che nella Roma antica tra il latino (latinitas, grammaticae artis) e il volgare (vulgare) la differenza fosse solo graduale. ${ }^{41}$

Si enim quod dixit etiam stabit, omnes pariter Latinis verbis usos, mulieres et viros, servos et liberos, doctos et litteratum ignaros; cum diverssam pro vitae et morum qualitate dicendi facultatem plurimis fuisse concesserim, eos qui domestica consuetudine et studiorum flagrantia elegantissimae orationis praestantiam, quod de Cesare supra est dictum, consecuti fuerint, maioribus quam quae possent a multitudine intellegi verbis uti debuisse...(B1. XV, 64)

Il latino forma una sorta di unità, però consiste di due varietà diverse, la prima usata dagli eruditi e l'altra dal popolo. Tale unità dei due idiomi viene espressa esplicitamente: "ut litterata orationis Latinitate, quam Romanis ... unicam fuisse" (B1. VII, 37). Biondo mette in evidenza che per quanto il latino e il volgare si differenzino molto, si tratta tuttavia di differenze graduali, dato che non è raro che i docti e gli illiterati utilizzino le stesse parole (omnes pariter latinis verbis usos, B1. XV, 64).

Sulla base della teoria secondo cui il volgare è imparentato con il latino della grammatica, dell'orazione e della letteratura, è possibile attribuire al volgare del popolo (basso) almeno una parziale grammaticalità, nel senso che questa varietà bassa dimostra di avere alcune categorie grammaticali (cfr. Coseriu e Meisterfeld, 2003: 155).

Tempora vero, modos numerosque et casus ab arte illas nequaquam nosse non dubito, quas tamen alicubi errantes multa recte et ordine video proferre...(Bl. XXII, 100)

Mentre Bruni ragiona dal punto di vista di un grammatico, Biondo assume la prospettiva di uno storico (cfr. Tavoni, 1984: 9) e di un retorico (cfr. Tavoni, 1982: 239). In qualità di retorico si ispira al modello che Cicerone ha presentato nei suoi lavori Orator, De Oratore e Brutus, e adotta i tre stili (genera dicendi), nonché le tre forme della lingua (tres latinae dictionis formas, B1. VIII, 39) per dichiarare la variabilità della lingua latina. ${ }^{42}$ Soprattutto come storico s'interessa allo sviluppo del volgare latino all'italiano, cioè al volgare contemporaneo. ${ }^{43} \mathrm{La}$ ragione principale per cui l'italiano è diverso dal latino, secondo lui è da ricercare nell'influenza corrompente dei Germani, soprattutto dei Goti e dei Vandali (adulterinam hanc barbarica mixtam loquelam habeamus vulgarem, B1. XXV, 110). ${ }^{44}$ Con questa spiegazione monocausale, Biondo diventa il fondatore della cosiddetta teoria della 
catastrofe (ingl. corruption of the Latin language, ted. Barbarenthese) (cfr. Marrazzini, 1993: 259-260; Ellena, 2011: 64-67). ${ }^{45}$

Temporibus vides quae Ciceronis aetatem praecesserant illos qui aut extra Romam vixerant, aut Romae domesticam habuerant aliquam barbariem, a nitore locutionis romanae aliqualiter recessisse, et barbarie illa infuscatos fuisse: postea vero quam urbs a Gothis et Vandalis capta inhabitarique coepta est, non unus iam aut duo infiscati, sed omnes sermone barbaro inquinati ac penitus sordidati fuerunt; sensimque factum est, ut pro romana latinitate adulterinam hanc barbarica mixtam loquelam habeamus vulgarem. (B1. XXV, 110-111)

Con l'attribuzione della grammaticalità al volgare, Biondo ha dissolto la dicotomia medievale ars vs. natura riguardo alle lingue (cfr. Ellena, 2011: 65), ${ }^{46}$ elemento che rappresenta un progresso importante nella cognizione linguistica, parimenti all'idea del mutamento linguistico, ossia lo sviluppo dall'antico volgare al moderno.

L'aspetto da chiarire è come Biondo si sia immaginato la corruzione del latino (corruptio), ${ }^{47}$ o più precisamente quali varietà si siano corrotte. Coseriu e Meisterfeld (2003: 158), che hanno rappresentato la loro interpretazione anche in un grafico, suggeriscono che Biondo credesse che il volgare contemporaneo (l'italiano del Quattrocento) si fosse sviluppato dal latino letterario e dal latino volgare allo stesso modo e che l'invasione dei Barbari avesse corrotto tutto il latino.

Tavoni invece non spiega veramente come Biondo intendesse il mutamento dal latino al volgare. È vero che Tavoni (1984: 19-22) è molto preciso riguardo alla "tripartizione della latinità" di Biondo, che è una tripartizione dapprima dipendente dal grado di grammaticalità (ars) secondo forma poetica, forma oratoria e forma vulgaris (dictionis formas, B1. VIII, 39), e poi dipendente piuttosto dal lessico secondo genus tenue/humile, genus medium/mediocre e genus grave/grande (genera dicendi, figura orationis), ${ }^{48}$ tuttavia non risulta chiaro quale varietà sia coinvolta nella corruzione barbarica e in quale modo.

Cum enim inde colligi liceat tres latinae dictionis formas tunc fuisse, poeticae unam numeris astrictam, oratoriae alteram nec contextam numeris nec carentem, vulgaris tertiam fluentem et quaquaversum sine numero sine ordine dilabentem; quae docti partes erant, quae indocti apparet. (B1. VIII, 39)

Sembra verosimile che Biondo ritenesse che fosse il volgare a "deteriorarsi" con le invasioni barbariche, dunque principalmente la forma vulgaris (probabilmente non la forma oratoria e la forma poetica), ossia lo stile minore (genus tenue), piuttosto che la grammatica (forse con la forma poetica e la forma oratoria), considerata da lui più o meno invariabile. ${ }^{49}$

Dobbiamo considerare che Biondo ha introdotto una prima idea della variabilità nella lingua latina, cioè ha formulato un approccio a un continuum diasistematico, grazie soprattutto a Cicerone e ai suoi concetti stilistici e retorici. I livelli retorici (genera dicendi e formae orationis) gli danno un'idea della diversità linguistica, perciò 


\section{Flavio Biondo}

De verbis romanae locutionis Blondi ad Leonardum Aretimum (1435)

omnes pariter Latinis verbis usos, mulieres et viros, servos et liberos, doctos et litteratum ignaros [...]

(Bl. XV, 64)

tres Latinae dictionis formas tumc fuisse, poeticae unam [...] oratoriae alteram [...] vulgaris tertiam [...]. (Bl. VIII, 39)

I'antichità

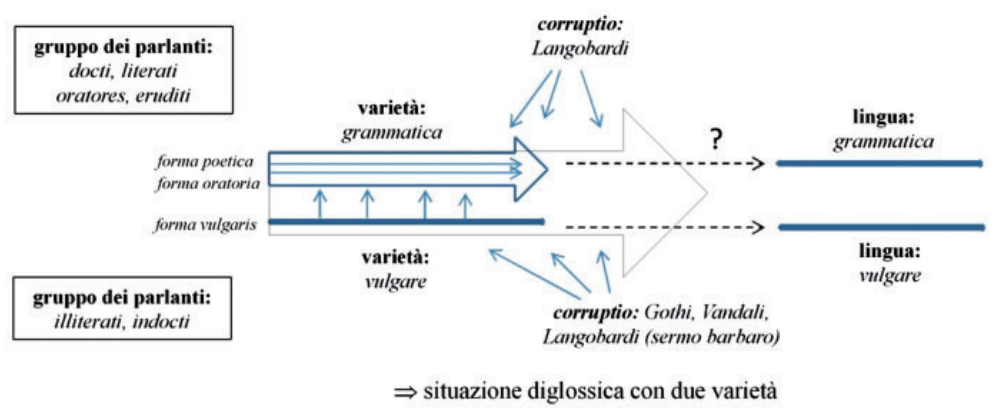

Figura 2. La situazione linguistica secondo Flavio Biondo.

è ancora lontano dal concetto di una vera varietà nel senso moderno. Per lui non era neanche concepibile il mutamento linguistico a tutti i livelli della lingua ${ }^{50} \mathrm{La}$ sua prospettiva potrebbe essere caratterizzata come quella di uno storico con un interesse linguistico sulla base della retorica. ${ }^{51}$

Per rendere più chiaro possibile il concetto linguistico di Biondo, abbiamo illustrato gli elementi più importanti nel grafico seguente:

Biondo dimostra una chiara unità di vedute con Bruni riguardo al gruppo dei parlanti, ossia a livello diastratico. In due aspetti decisivi, però, Biondo si distingue da Bruni: riconosce che il latino antico consiste di forme diverse (sorte di varietà diafasiche e diastratiche) che tuttavia formano un'unità linguistica. Inoltre sostiene che il volgare (l'italiano) contemporaneo si è sviluppato dal latino antico, pur non precisando esattamente da quale forma di latino (da cui il punto interrogativo nel grafico). Egli dunque prende in considerazione un mutamento linguistico che per Bruni non era invece al centro dell'attenzione.

\section{Conclusione}

Un problema generale dell'interpretazione adeguata dei modelli di Bruni e Biondo (e anche di Dante) è che sembra arbitrario suggerire che i suddetti scritti trattino problemi o questioni della linguistica moderna, perché l'intenzione era rispettivamente diversa. Invece è lecito applicare una terminologia scientifica moderna per capire meglio le idee e i concetti che prefigurano la nozione attuale della lingua. 
Con questa premessa abbiamo cercato di mostrare in che modo il dibattito tra Leonardo Bruni e Flavio Biondo abbia apportato innovazioni nella storia della coscienza linguistica, e in quali aspetti fosse invece ancora radicato nel medioevo.

Anche se la ricezione del trattato De vulgari eloquentia era marginale prima della "riscoperta" di Trissino, tale opera è centrale per comprendere la cognizione linguistica di Dante e rappresenta egregiamente il patrimonio intellettuale del medioevo riguardo al latino. Per questo è anche essenziale per capire poi le teorie di Bruni e Biondo.

Oltre al fatto dell'invariabilità della lingua latina, Dante è molto moderno nella sua concezione dei molteplici aspetti della variabilità del volgare e del mutamento linguistico.

Bruni e Biondo, invece, portano la prima volta il discorso sulla situazione linguistica dell'antichità e il carattere del latino antico. In linea di principio entrambi sono d'accordo sul fatto che esisteva nella Roma antica un latino letterario regolato da una grammatica, parlato e scritto dai colti, e un latino volgare per la comunicazione di ogni giorno del popolo, chiaramente meno regolata e "rozza". Tuttavia Bruni insiste sulle grandi differenze tra latino e volgare nel senso di una relazione diglossica, soprattutto citando l'argomento dantesco della grammaticalità del latino (ars grammatica, latinitas), mentre Biondo con l'aiuto della retorica illustra verosimilmente la situazione linguistica dell'antichità romana e dà anche una prima idea di un "latino volgare". Nel caratterizzare il latino come lingua diasistematicamente diversificata, Biondo esprime un concetto in un certo qual modo convergente con l'idea del volgare di Dante.

\section{Note}

1. La prima attestazione del sermo vulgaris si trova tuttavia nella Rhetorica ad Herennium (cfr. Rhet. ad Her. IV, 56 (69)), testo anonimo che è anche stato attribuito a Cicerone (cfr. Müller, 2001: 155-156) il quale ha divulgato la nozione sermo vulgaris, l'unico riferimento dei dotti rinascimentali. Si veda p.es. il paragrafo seguente: "didicisti enim non posse nos Amalfinii aut Rabirii similes esse, qui nulla arte adhibita de rebus ante oculos positis vulgari sermone disputant" (Cic. Acad. I, 5). Per una breve storia della nozione latino volgare (ingl. vulgar Latin, ted. Vulgärlatein) cfr. Kiesler (2006: 3-14) e Lloyd (1979: 111-112).

2. Certamente il latino del medioevo non era una lingua morta come ai giorni nostri, però non era più neanche una lingua con native speakers; per una discussione intorno al latino come lingua viva, lingua mezza viva e lingua morta affatto cfr. Lüdtke (2005: 40-42) e Faithfull (1953: 278-281).

3. Siccome questo testo di Dante è lo sfondo decisivo per la discussione che segue nel Quattrocento, abbiamo concesso un po' più di spazio al capitolo corrispondente, in definitiva anche perché si tratta di un testo molto complesso.

4. "Nel corso del Medioevo l'opera dantesca fu solo menzionata in maniera vaga, senza una conoscenza diretta del testo. Ad essa accennano Giovanni Villani, Antonio Pucci, e soprattutto Giovanni Boccaccio. La stessa scarsità dei manoscritti del De vulgari (sono tre in tutto quelli anteriori al XVI secolo) dimostra che il testo circolò pochissimo" 
(Marazzini, 1993: 237). Questo nonostante il trattato sia importante per capire la mentalità medievale, l'innovazione dantesca e in seguito le esposizioni di Bruni e Biondo.

5. Per un confronto gramatica vs. vulgare si veda p.es. Mengaldo (1971: 656-658), Ineichen (1973: 69-72), Cecchin (1988: X-XII).

6. Tavoni (1987: 397) obietta che Dante parla del latino sempre come locutio e non come lingua, in modo da non precisare esattamente il legame tra gramatica e vulgare.

7. Si veda Aegidius Romanus (1243/1247-1316) che in De regimine principum (cfr. De reg. princ. lib. II, pars II, cap. 7) parla del latino come una lingua creata per i filosofi: "Videntes enim Philosophi nullum idioma vulgare esse completum \& perfectum, per quod perfecte exprimere possent naturas rerum, \& mores hominum, \& cursus astrorum, $\&$ alia de quibus disputare volebant, invenerunt sibi quasi proprium idioma, quod dicitur latinum, vel idioma literale: quod constituerunt adeò latum \& copiosum, ut per ipsum possent omnes suos conceptus sufficienter exprimere" (1607: 304).

8. Dante stesso cita la lingua dei Greci (quidem secundariam Greci habent, DVE I, 1,3) e altre lingue simili (et alii, DVE I, 1, 3). In DVE (2007: 23, nota 6) Frings e Kramer suppongono che sia forse l'arabo, Imbach e Suarez-Nani (2007: 75) sostengono che non è dimostrato che cosa significhi et alii, però le interpretazioni più frequenti indicano gli Arabi e gli Ebrei.

9. In analogia alla distinzione di Aristotele ('A

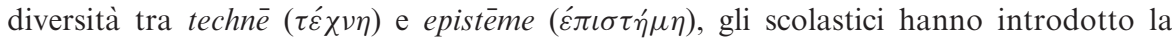
differenziazione tra ars e scientia, cioè con l'inizio della grammatica speculativa la grammatica è rivalutata, dunque diventa anche scientia, non solo ars nel senso di una capacità tecnica (cfr. Jungen e Lohnstein, 2007: 93). Per un breve sommario delle prime grammatiche volgari dell'area romanza circa la nozione "grammatica" cfr. Schäfer-Prieß (2000: 63-66).

10. "In verborum declinationibus disciplina loquendi dissimilitudinem an similitudinem sequi deberet, multi quaesierunt. Cum ab his ratio quae ab similitudine oriretur vocaretur analogia, reliqua pars appellaretur anomalia" (Varr., De ling. lat. X, 1).

11. "Declinatiionum genera sunt duo, voluntarium et naturale; voluntarium est, quo ut cuiusque tulit voluntas declinavit" (Varr., De ling. lat. VIII, 21).

12. Il principio dell'analogia si mostra soprattutto nella flessione (declinatio naturalis) e il principio dell'anomalia nella formazione delle parole (declinatio voluntaria). Nuove parole non possono solo nascere tramite derivazione (e composizione), ma anche tramite una creazione nuova (impositio); spesso impositio e declinatio voluntaria sono congruenti. Forse Varrone prova a risolvere come arbitror la disputa tra analogisti (scuola di Alexandria) e anomalisti (scuola di Pergamon). Tale discussione viene principalmente riferita solo da Varrone (cfr. Ax, 2000: 146; Grebe, 2000: 199-201).

13. Con l'andare del tempo anche gli insegnanti (grammatici) sono tenuti in tale onore che a loro stessi è attribuito lo stato di auctoritas dai discipuli e dalla società intellettuale, forse anche in associazione con un aspetto morale (mores) (cfr. Poccetti et al., 2005: 419).

14. Per il latino come grammatica all'epoca di Dante - ad esempio in Guido de Columnis (XIII sec.) nella cui Historia destructionis Troiae (1287) si trova il passo qui gramaticam legunt (1936: 4, f. I ${ }^{\mathrm{V}}$ ) - cfr. Curtius (1943: 161-162).

15. Riguardo alla variabilità del volgare si vedano anche il noto passo nella Divina Commedia (Div. Comm., Par. XXVI 114, 130-132) e passim Il Convivio, ad esempio "lo volgare seguita uso, e lo latino arte" (Conv. I, 5, 14). Per un'analisi linguistica del Convivio cfr. Bossong (1990: 44-50). 
16. Cfr. p.es. Ineichen (1973: 75-77), Wunderli (1994: 95-107), Ellena (2004: 75-77) o Pötters (2005: 391-393).

17. "Hec est nostra vera prima locutio" (DVE I, 2, 1).

18. La differenziazione delle lingue attraverso la particella dell'affermazione, un criterio minimalistico (cfr. le carte e tavole da Ternes, 1989: 66, 69, 71-73) che risulta in un primo e un secondo ydioma tripharium, non solo stabilisce una distribuzione tipologica (come è detto spesso, cfr. p.es. Schmitt, 1975: 312-315), ma anche una distribuzione genetica e areale: genetica, perché deduce le lingue da una proto-lingua, e areale, perché aggiunge una chiara descrizione geografica dei limiti linguistici in Europa. Inoltre aggiunge l'aspetto della migrazione (DVE I, 8, 2) che è essenziale per la Ausdifferenzierung delle lingue indoeuropee e più tardi per le lingue romanze. L'intuizione giusta di Dante della migrazione indoeuropea è solo bloccata dalla sua immaginazione cristiana, cioè dalle storie bibliche.

19. "Quare ad minus xiiii vulgaribus sola videtur Ytalia variari" ( $D V E \mathrm{I}, 10,7)$. Questo è il suo risultato dopo aver enumerato tutti i dialetti dell'italiano a destra e a sinistra dell'Appennino (iugum Apenini, DVE I, 10, 4). Il criterio del Latium bipartitum non è quello della linguistica moderna, però quando si visualizzano i confini dei dialetti non è così erroneo (cfr. tuttavia Coseriu e Meisterfeld (2003: 133) che dicono esplicitamente falsch).

20. Per una discussione dei singoli dialetti e le loro caratteristiche menzionate da Dante cfr. Holtus (1989: 5-8), e per un grafico con i dialetti e la tipologia delle lingue cfr. Holtus (1987: 349).

21. Osserva molto chiaramente che c'è una differenza tra la variazione fra due dialetti come il lombardo (a Milano) e il veneziano (a Verona) oppure il romano (a Roma) e il toscano (a Firenze) e la variazione all'interno di un dialetto come tra il vernacolo a Napoli e a Gaeta oppure tra quello a Ravenna e a Faenza: "et quare vicinus habitantes adhuc discrepant in loquendo, ut Mediolanses et Veronenses, Romani et Florentini, nec non convenientes in eodem genere gentis, ut Neapolitani et Caetani, Ravennates et Faventini" (DVE I, 9, 4). Per esemplificare la differenza linguistica tra due quartieri sceglie il Borgo San Felice e il quartiere della Strada Maggiore a Bologna (Bononiensis Burgi Sancti Felicis et Bononiensis Strate Maioris, DVE I, 9, 4).

22. Questo si può dedurre dal fatto che in una città medievale i cittadini si raggruppano secondo il loro mestiere e anche secondo la loro posizione nella società - le due componenti non sono veramente da distinguere. Wunderli (1994: 102-103) ad esempio lo interpreta solo come diastratico, però parla anche di Fachsprachen.

23. "Solis etenim in uno convenientibus actu eadem loquela remansit: puta cunctis architecoribus una, cunctis saxa volventibus una, cunctis ea parantibus una; et sic de singulis operantibus accidit" (DVE I, 7, 7). Ovviamente Dante ha potuto osservare che i diversi gruppi professionali hanno un vocabolario specifico, un modo proprio di parlare (da notare sempre anche un aspetto diastratico).

24. "et quanto excellentius exercebant, tanto rudius nunc barbariusque locuntur" (DVE I, $7,7)$. In questo caso è interessante notare che la loquela è tanto più rozza e più bassa quanto più alto è invece il prestigio della professione, inversamente alla prospettiva normale. In questo caso il mito di Babele funziona come esempio per un disordine linguistico, però risuona forse anche un aspetto cristiano, morale.

25. Wunderli (1994: 103-104) non è chiaro rispetto al livello teorico, perché assume i due fenomeni sotto la diamesica, cioè scritto (latino) vs. parlato (volgare) in $D V E$ e scritto vs. parlato nel volgare, descritto nella Vita Nuova (XXV, 7). 
26. Si veda anche la variabilità descritta nel Convivio: "Onde vedemo ne le cittadi d'Italia, se bene volemo agguardare, da cinquanta anni in qua molti vocabuli essere spenti e nati e variati; onde se 'l picciol tempo così trasmuta, molto più trasmuta lo maggiore. Sì ch'io dico, che se coloro che partiron d'esta vita già sono mille anni tornassero a le loro cittadi, crederebbero la loro cittade essere occupata da gente strana, per la lingua da loro discordante" (Conv. I, 5, 9).

27. "Ein Phänomen der dritten Art ist die kausale Konsequenz einer Vielzahl individueller intentionaler Handlungen, die mindestens partiell ähnlichen Intentionen dienen” (Keller, 1994: 92).

28. Sembrano due le ragioni per cui Dante non è esplicito in questo paragrafo: da una parte ha una buona intuizione che ci debba essere una specie di proto-lingua, però non è sicuro riguardo al suo carattere; dall'altra parte l'ydioma tripharium ha un aspetto mitico che non si deve chiarire.

29. "ut silvae foliis pronos mutantur in annos, / prima cadunt: ita verborum vetus interit aetas, / et iuvenum ritu florent modo nata vigentque.../ multa renascentur quae iam cecidere cadentque / quae nunc sunt in honore vocabula, si volent usus, / quem penes arbitrium est et ius et norma loquendi" (Oraz., Ars poet., 60-63, 70-72). Citato ad esempio da Bossong (1990: 57-58) o da Ellena (2004: 76), entrambi non in chiaro sul punto che per Dante non può essere il latino, che è variabile.

30. A causa dei disordini a Roma, il papa tra il 1434 e il 1443 ebbe il suo domicilio al di fuori della città sacra, prima a Firenze, poi a Bologna e Ferrara e dopo di nuovo a Firenze (Coseriu e Meisterfeld, 2003: 149).

31. Le posizioni dei singoli eruditi in questo dibattito erano le seguenti: "Mentre, infatti, Biondo Flavio, Poggio Bracciolini e Andrea ritenevano che tutta la popolazione nell'antichità avesse parlato la medesima lingua, Cencio Rustici e Antonio Loschi ipotizzavano l'esistenza di due differenti lingue adoperate rispettivamente dai dotti e dagli indotti"' (Marcellino e Ammannati, 2015: 3).

32. Cronologicamente il primo documento fu quello di Biondo (1 aprile 1435) e il secondo quello di Bruni (7 maggio 1435) (cfr. Tavoni, 1984: 3, nota 1). Tuttavia dapprima trattiamo Bruni, data la sua posizione più conservativa.

33. "La disputa, nel corso dell'incontro fiorentino, dovette dunque avere il carattere di una contrapposizione non tra ragionamenti collegati in una solida costruzione concettuale, ma piuttosto tra punti di vista resi inconciliabili perché dettati da un diverso modo di rappresentarsi la consuetudine linguistica degli antichi sul fondamento della prassi linguistica odierna" (Marchiò, 2008: 21).

34. Per informazioni biografiche e opere ulteriori di Leonardo Bruni (p.es. Historia del popolo fiorentino, De interpretatione recta, Vite parallele di Dante e Petrarca, Dialogi ad Petrum Paulum Histrum, De studiis et litteris) e di Flavio Biondo (p.es. Historiarum ab inclinatione Romani imperii Decades 3, Roma instaurata, Italia illustrata, Roma triumphans) cfr. Coseriu e Meisterfeld (2003: 150).

35. "Atque Latina lingua a vulgari in multis differt, plurimum tamen terminatione, inflexione, significatione, constructione et accentu, de quibus omnibus simul dicamus: nam seorsum prosequi singula longum foret" (Br. 32).

36. Generalmente bisogna essere prudenti nell'interpretazione dell'opinione esatta di Bruni, perché tranne la sua breve lettera non esiste un altro suo trattato su questo soggetto e una grande parte della discussione è trasmessa solo attraverso il trattato più dettagliato del suo rivale Biondo. 
37. Per la distribuzione tra latino e volgare (italiano) nella produzione letteraria in Italia del Trecento e del Quattrocento cfr. Kristeller (1984).

38. Il grafico di Coseriu e Meisterfeld (2003: 158) suggerisce che Bruni abbia postulato che il volgare antico sia diventato il volgare della sua epoca (senza mutamento linguistico). Perciò si tratta di un'interpretazione che non è contenuta letteralmente nel testo. Bruni non esprime chiaramente come intenda la relazione tra i due volgari, in quanto tale aspetto non è al centro della sua indagine.

39. Bruni non distingue chiaramente tra il livello diafasico e il livello diastratico. Di più, per lui, la complessità delle strutture grammaticali è strettamente legata a un registro alto, che dal punto di vista moderno non è così per forza di cose (cfr. Blanche-Benveniste, 2005: 57-62; Kiesler, 2013: 259-260).

40. Marcellino e Ammannati (2015: 22) rilevano giustamente che Bruni non adduce tanti argomenti a sostegno di questa differenza: "da un punto di vista linguistico, infatti, ebbe pochissimi elementi per ipotizzare una netta demarcazione tra lingua dei dotti e quella degli indotti nell'antica Roma."

41. Riguardo alla terminologia Biondo segue l'uso medievale (e così Dante), perché usa il termine "grammatica" come sinonimo di "latino" (cfr. B1. I, 8: "nostra vulgato idiomate, an grammaticae artis usu, quod Latinum appellamus"; Bl. II, 13: "in grammaticam Latinitatem").

42. Già Cicerone distingue i tre stili e le forme dell'orazione o più precisamente di una Diskurstradition (it. "tradizione del discorso", ingl. "discourse tradition"; cfr. Wilhelm, 2001: 467-468): "Tria sunt omnino genera dicendi" (Cic., Orat., 5, 20) vs. "Sed quoniam plura sunt orationum genera caque diversa neque in unam formam cadunt omnia" (Cic., Orat., 11, 37).

43. Tuttavia, la prospettiva di Biondo (e di Bruni) non è quella di un linguista: "Gli umanisti, evidentemente, non erano interessati a ricercare le origini della lingua moderna, ma scoprivano queste origini cercando di metter meglio a fuoco le cause della crisi della romanità, esplorando la frattura che aveva dato inizio al medioevo" (Marazzini, 1993: 240).

44. Nel suo lavoro successivo Italia illustrata (1474) Bruni rivede le proprie posizioni e considera i Longobardi come i responsabili principali della corruzione del latino (cfr. Klein, 1957: 57). Anche Dante aveva già una vaga idea dell'invasione barbarica e della sua possibile influenza sulla lingua, seppur in modo non sistematico: "hanc ex commixtione advenarum Longobardorum terrigenis credimus remansisse" (DVE I, 15, 3).

45. Per un riflesso della teoria della catastrofe e la tesi di una corruptio del latino causata dai barbari in altri paesi come Spagna, Francia, Inghilterra e Germania cfr. Neis (2009: 574-579).

46. Tavoni (1984: 22) precisa che per Biondo è piuttosto un continuum, cioè "gradi diversi di ars".

47. Per il concetto di corruzione secondo Aristotele, che in De generatione et corruptione

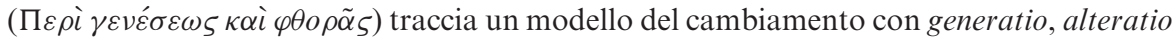
e corruptio, cfr. Schunck (2003: 17-18).

48. I tre stili sono menzionati esplicitamente da Biondo: p.es. "dicendi genere oratores" (B1., XV, 65), "tria ipsa dicendi genera" (B1. XVII, 72), "figuris orationis...gravi ... mediocri... attenuata" (B1. XVII, 72). Importante è la sua trattazione del medius modus (Bl. XVI, 68-71), laddove esprime l'idea che l'oratore deve cambiare il suo modo di parlare quando si indirizza al popolo - un modo di parlare indipendente dai 
tre stili con un lessico adattato alle esigenze del pubblico; cfr. anche Fubini (1961: 534), Tavoni (1984: 22-23) e Delle Donne (2008: XXVI).

49. Si veda anche la posizione di Mazzocco (1993: 49): "Biondo also judges the nature of classical Latin solely in terms of social differences (urbanitas vs. rusticitas), disregarding almost completely the changes of speech due to time and space."

50. Cfr. anche Fubini (1961: 535) il quale dichiara che la distinzione di Biondo "rimane provvisoria e schematica".

51. Mazzocco (1993: 40-42) dichiara come l'interesse storico fu introdotto nel discorso intellettuale del Rinascimento, quasi come presupposto della disputa fra Bruni e Biondo. Tuttavia è forse eccessivo caratterizzare il lavoro di Biondo come "linguistic archeology" (42) e attribuirgli "dispassionate concern for truth", e quello di Bruni guidato solo da "utilitarian goals" (42). Prima di tutto è importante precisare che neanche Biondo era un linguista nel senso moderno e, in secondo luogo, che anche lui aveva un interesse particolare in questo dibattito.

\section{Bibliografia}

\section{Fonti primarie:}

Aegidius Romanus (Aeg. Rom.), De reg. princ. = Colonna, Egidio (Romanus, Aegidius) (1607) De regimine principum libri III. Recogniti et una cum vita auctoris in lucem editi per F. Hieronymum Samaritanium. Roma: Bartholomaeum Zannettum.

Rhet. ad Her. = Auctor ad Herennium (1994) Rhetorica ad Herennium. Lateinisch-Deutsch. A cura e traduzione tedesca di T Nüßlein. Zurigo: Artemis \& Winkler.

Biondo (B1.) = Flavio Biondo (1984) De verbis Romanae locutionis Blondi ad Leonardum Aretinum. In: Tavoni M (a cura di) Latino, grammatica, volgare: Storia di una questione umanistica. Padova: Antenore, pp. 197-215.

Bruni (Br.) = Leonardo Bruni (Leonardo Aretino) (1984) Leonardus Flavio Forolivensi S. Quaerit an vulgus et literati eodem modo per Terentii Tullique tempora Romae locuti sint. In: Tavoni M (a cura di) Latino, grammatica, volgare: Storia di una questione umanistica. Padova: Antenore, pp. 216-221.

Cicerone (Cic.), Orat. = Marcus Tullius Cicero (1988) Orator. Lateinisch-Deutsch. A cura di B Kytzler. Monaco di Baviera-Zurigo: Artemis.

Cicero (Cic.), Acad. = Marcus Tullius Cicero (1990) Hortensius, Lucullus: Academici libri. Lateinisch-Deutsch. A cura, traduzione tedesca e commenti di L Straume-Zimmermann, F Broemser e O Gigon. Monaco di Baviera-Zurigo: Artemis.

Dante, Div. Comm. = Dante Alighieri (1988) Die Göttliche Komödie. Italienisch und Deutsch. 6 voll. Traduzione tedesca e commenti di H Gmelin. Monaco di Baviera: Deutscher Taschenbuch Verlag.

Dante, Vita Nuova = Dante Alighieri (1988) Vita Nuova. Das neue Leben. Traduzione tedesca e commenti di A Coseriu A e U. Kunkel. Monaco di Baviera: Deutscher Taschenbuch Verlag.

Dante, Conv. = Dante Alighieri (1996) Das Gastmahl, I. Italienisch-Deutsch. Traduzione tedesca di T Ricklin, introduzione e commenti di F Cheneval. Amburgo: Meiner.

Dante, DVE = Dante Alighieri (2007) De vulgari eloquentia. Traduzione italiana di GG Trissino (1529); traduzione tedesca di M Frings e J Kramer. Stoccarda: ibidem.

Guido de Columnis, Hist. destr. = Guido de Columnis (1936) Historia desctructionis Troiae. A cura di NE Griffin. Cambridge: Medieval Academy of America. 
Orazio, Ars poet. = Quintus Horatius Flaccus (1984) Ars poetica. Die Dichtkunst. LateinischDeutsch. A cura e traduzione tedesca di E Schäfer. Stoccarda: Reclam.

Quintiliano (Quint.), Inst. orat. = Marcus Fabius Quintilianus (2001) The Orator's Education, Books 1-2. A cura e traduzione inglese di DA Russel. Cambridge (Mass.): Harvard University Press.

Varrone (Varr.), De ling. lat. = Marcus Terentius Varro (1958) On the Latin Language, II, Books 8-10. Traduzione inglese di RG Kent. Cambridge (Mass.): Harvard University Press, London: Heinemann.

\section{Fonti secondarie:}

Ax W (2000) "Disputare in utramque partem": Zum literarischen Plan und zur dialektischen Methode Varros in de lingua Latina 8-10. In: Ax W e Grewing F (a cura di) Lexis und Logos: Studien zur antiken Rhetorik. Stoccarda: Steiner, pp. 140-163.

Berruto G (1987) Sociolinguistica dell'italiano contemporaneo. Roma: Nuova Italia Scientifica.

Berruto G (2003) Fondamenti di sociolinguistica. Roma, Bari: Laterza.

Blanche-Benevniste C (2005) De la spécifité de l'oral. In: Van Deyck R, Sornicola R e Kabatek J (a cura di) La variabilité en langue. Vol. 2: Les quatres variations. Gent: Communication \& Cognition, pp. 45-64.

Bossong G (1990) Sprachwissenschaft und Sprachphilosophie in der Romania: Von den Anfängen bis August Wilhelm Schlegel. Tübingen: Narr.

Cecchin S (1988) Introduzione. In: Cecchin S (a cura di) De vulgari eloquentia. Milano: TEA, pp. I-XV.

Cheneval F (1996) (a cura di) Das Gastmahl, I. Italienisch-Deutsch. Traduzione tedesca di T Ricklin, introduzione e commenti di F Cheneval. Amburgo: Meiner.

Coseriu E (1973) Probleme der strukturellen Semantik: Vorlesung gehalten im Wintersemester 1965/66 an der Universität Tübingen. A cura di D Kastovsky. Tübingen: Narr.

Coseriu E and e Meisterfeld R (2003) Geschichte der romanischen Sprachwissenschaft. Vol. 1. Von den Anfängen bis 1492: Tübingen: Narr.

Curtius ER (1943) Dante und das lateinische Mittelalter (Mittelalter-Studien, XX). Romanische Forschungen 57: 151-185.

Delle Donne F (2008) Introduzione. In: Delle Donne F (a cura di) Flavius Blondus: De verbis Romanae locutionis. Roma: Istituto storico italiano per il Medio Evo, pp. XV-LVII.

Diez F (1870) Grammatik der romanischen Sprachen. Erster Theil. Bonn: Weber.

Ellena S (2004) “Opera naturale è ch'uom favella": Dantes Sprachtheorie. Italienisch 51: $70-94$.

Ellena S (2011) Die Rolle der norditalienischen Varietäten in der "questione della lingua": Eine diachrone Untersuchung zu Sprachbewusstsein, Sprachwissen und Sprachbewertung. Berlino-Boston: de Gruyter.

Faithfull GR (1953) The Concept of "Living Language" in Cinquecento Vernacular Philology. The Modern Language Review 48: 278-292.

Ferguson CA (1959) Diglossia. Word 15: 325-340.

Fubini R (1961) La coscienza del latino negli umanisti: “An latina lingua Romanorum esset peculiar idioma". Studi medievali II(2): 505-550.

Grebe S (2000) Kriterien für Latinitas bei Varro und Quintilian. In: Haltenhoff A e Mutschler FH (a cura di) Hortus Litterarum Antiquarum: Festschrift für Hans Arnim Gärtner zum 70. Geburtstag. Heidelberg: Winter, pp. 191-210. 
Holtus G (1987) Zur Sprach- und Wortgeschichte von "latino" und "volgare" in Italien. In: Dahmen W et al. (a cura di) Latein und Romanisch: Romanistisches Kolloquium I. Tübingen: Narr, pp. 340-354.

Holtus G (1989) Das “vulgare illustre" als Modell einer italienischen Kunstsprache: Standard, Substandard und Varietät in Dante Alighieris Traktat "De vulgari eloquentia" (1305). In: Holtus G e Radtke G (a cura di) Sprachlicher Substandard II: Standard und Substandard in der Sprachgeschichte und in der Grammatik. Tübingen: Niemeyer, pp. 1-13.

Imbach R e Suarez-Nani T (2007) Kommentar. In: Imbach R e Suarez-Nani T (a cura di) Dante Alighieri: Über die Beredsamkeit in der Volkssprache. Lateinisch-Deutsch. Traduzione tedesca di F Cheneval, introduzione di R Imbach e I Rosier-Catach I, commenti di R Imbach e T Suarez-Nani T. Amburgo: Meiner, pp. 67-155.

Ineichen G (1973) Das Verhältnis Dantes zur Sprache. Deutsches Dante-Jahrbuch 48: 63-78. Jungen O e Lohnstein H (2007) Geschichte der Grammatiktheorie: Von Dionysios Thrax bis Noam Chomsky. München: Fink.

Keller R (1994) Sprachwandel: Von der unsichtbaren Hand in der Sprache. Tübingen-Basilea: Francke.

Kiesler R (2006) Einführung in die Problematik des Vulgärlateins. Tübingen: Niemeyer.

Kiesler R (2013) Zur Syntax der Umgangssprache: Vergleichende Untersuchungen zum Französischen, Italienischen und Spanischen. Darmstadt: Wissenschaftliche Buchgesellschaft.

Klein HW (1957) Latein und Volgare in Italien: Ein Beitrag zur Geschichte der italienischen Nationalsprache. München: Hueber.

Koch P e Oesterreicher W (1985) Sprache der Nähe - Sprache der Distanz: Mündlichkeit und Schriftlichkeit im Spannungsfeld von Sprachtheorie und Sprachgeschichte. Romanistisches Jahrbuch 36: 15-43.

Koch P e Oesterreicher W (2011) Gesprochene Sprache in der Romania: Französisch, Italienisch, Spanisch. Tübingen: Niemeyer.

Kristeller PO (1984) Latein und Vulgärsprache im Italien des 14. und 15. Jahrhunderts. Deutsches Dante-Jahrbuch 59: 7-35.

Lüdtke H (2005) Der Ursprung der romanischen Sprachen: Eine Geschichte der sprachlichen Kommunikation. Kiel: Westensee.

Lloyd PM (1979) On the Definition of Vulgar Latin: The Eternal Return. Neuphilologische Mitteilungen 80: 110-122.

Marazzini C (1993) La speculazione linguistica nella tradizione italiana: Le teorie. In: Serianni L e Trifone P (a cura di) Storia della lingua italiana, I. I luoghi della codificazione. Torino: Einaudi, pp. 231-329.

Marazzini C (2013) Da Dante alle lingue del Web. Otto secoli di dibattiti sull'italiano. Roma: Carocci.

Marcellino G e Ammannati G (2015) Il latino e il "volgare" nell'antica Roma: Biondo Flavio, Leonardo Bruni e la disputa umanistica sulla lingua degli antichi Romani. Pisa: Edizioni della Normale.

Marchiò MB (2008) Come discutevano gli umanisti: Una disputa quattrocentesca sulla lingua parlata dai romani antichi. Firenze: Firenze Atheneum.

Mazzocco A (1993) Linguistic Theories in Dante and the Humanists: Studies of Language and Intellectual History in late Medieval and Early Renaissance Italy. Leiden: Brill.

Mengaldo PV (1971) Lingua. In: Istituto della Enciclopedia Italiana (a cura di) Enciclopedia Dantesca. Roma: Istituto della Enciclopedia Italiana, pp. 655-664. 
Müller R (2001) Sprachbewußtsein und Sprachvariation im lateinischen Schrifttum der Antike. Monaco di Baviera: Beck.

Neis C (2009) Korruption. In: Haßler G e Neis C (a cura di) Lexikon sprachtheoretischer Grundbegriffe des 17. und 18. Jahrhunderts, I. Berlino-New York: de Gruyter, pp. 567-582.

Poccetti P et al. (2005) Eine Geschichte der lateinischen Sprache: Ausformung, Sprachgebrauch, Kommunikation. Traduzione tedesca di H Bertsch. Tübingen-Basilea: Francke.

Pötters W (2005) Dantes Theorie des Sprachwandels von De vulgari eloquentia I zu Paradiso XXVI. In: Horiot B et al. (a cura di) Mélanges offerts au professeur Lothar Wolf: "Je parle, donc je suis... de quelque part”. Lyon: Centre d'Études linguistiques Jacques Goudet, pp. 385-407.

Schäfer-Prieß B (2000) Die portugiesische Grammatikschreibung von 1540-1822: Entstehungsbedingungen und Kategorisierungsverfahren vor dem Hintergrund der lateinischen, spanischen und französischen Tradition. Tübingen: Niemeyer.

Schmitt C (1975) Sprachtyplogie und Mundartforschung. Zeitschrift für Romanische Philologie 91: 310-338.

Schuchardt H (1866) Der Vokalismus des Vulgärlateins, I. Leipzig: Teubner.

Schunck M (2003) Der Sprachwandel im metalinguistischen Diskurs Italiens und Frankreichs von der Renaissance zur Aufklärung. Francoforte s.M.: Lang.

Tavoni M (1982) The 15th-Century Controversy on the Language Spoken by the Ancient Romans: An Inquiry into Italian Humanist Concepts of "Latin", "Grammar", and "Vernacular". Historiographia linguistica 9: 237-264.

Tavoni M (1984) Latino, grammatica, volgare: Storia di una questione umanistica. Padova: Antenore.

Tavoni M (1987) Contributo all'interpretazione di De vulgari eloquentia I, 1-9. Rivista di letteratura italiana 5: 386-453.

Ternes E (1989) La classification des langues romanes d'après Dante reconsidérée aujourd'hui. In: Section XIV: Histoire de la linguistique et de la philologie romanes. Section XV: Philologie romane et langues romanes: prise de conscience ou: la philologie pour quoi faire? Section XVI: Travaux en cours. Tübingen: Niemeyer, pp. 64-79.

Wilhelm R (2001) Diskurstraditionen. In: Haspelmath M et al. (a cura di) Language Typology and Language Universals: An International Handbook, I. Berlino-New York: de Guyter, pp. 467-477.

Wunderli P (1994) Dante - ein Linguist. Dante-Jahrbuch 68/69: 81-126. 